\title{
Exact Interference of Adaptive Variable-Rate Tasks under Fixed-Priority Scheduling
}

\author{
Alessandro Biondi, Alessandra Melani, Mauro Marinoni, Marco Di Natale, Giorgio Buttazzo \\ Scuola Superiore Sant'Anna, Pisa, Italy
}

\begin{abstract}
Engine control applications require the execution of tasks activated in relation to specific system variables, such as the crankshaft rotation angle. To prevent possible overload conditions at high rotation speeds, such tasks are designed to vary their functionality (hence their computational requirements) for different speed ranges. Modeling and analyzing such a type of tasks poses new research challenges in the schedulability analysis that are now being addressed in the real-time literature. This paper advances the state of the art by presenting a method for computing the exact worst-case interference of such adaptive variable-rate tasks under fixed priority scheduling, enabling a tight analysis and design of engine control applications.
\end{abstract}

\section{Introduction}

A large variety of real-time task models have been proposed in the literature to analyze the schedulability of different types of embedded systems. The classical Liu and Layland periodic task model [13] captures the typical structure of control loops providing an implementation for discrete-time controllers. The sporadic task model introduced by Mok [14] captures the intrinsic irregular arrival sequence of external events, while providing a bound on the worst-case arrival rate necessary to derive an offline schedulability analysis.

A rate-based execution abstraction was introduced by Jeffay et al. [10], [11] to generalize the classical periodic and sporadic scheme. According to such a model, a task specifies its expected rate as the maximum number $x$ of executions expected to be requested in any interval of length $y$, however the maximum computation time required for any job of the task is fixed, while the actual distribution of events in time is arbitrary.

The multi-frame model proposed by Mok and Chen [15] provides the additional expressivity to capture conditional executions and execution patterns. In this model, tasks are activated periodically, but the execution time of each job varies according to a predefined pattern. Such a model has been later generalized by Baruah et al. [2] to allow jobs to be separated by a varying interarrival time. However, in both cases the activation pattern is known a priori and does not depend on any state variable.

An elastic model has been presented by Buttazzo et al. [3], [5] to tolerate and handle permanent overload conditions in periodic real-time systems. According to this model, a task has a fixed computation time, but a variable period, which can be varied in a given range. An overload condition is then handled by properly compressing task utilizations as if they were elastic springs with given elastic coefficients, expressing the capability of each task to change its period.

More recently, the consideration of a fuel injection systems, as representatives of a possibly larger class of applications, has highlighted the limitations of the existing approaches and the need for a new type of task model and analysis.

The general goal of a fuel injection system is to determine the point(s) in time and the quantity of fuel to be injected in the cylinders of an engine, relative to the position of each piston, which is in turn a function of the angular position of the crankshaft. In a reciprocating engine, the dead centre is the position of a piston in which it is farthest from, or nearest to, the crankshaft. The former is the top dead centre (TDC) while the latter is the bottom dead centre (BDC), as illustrated in Figure 1. In a four-cylinder engine, the pistons are paired in phase opposition, so that, when two of them are in a TDC, the others are in a BDC. The TDC is the typical reference point, in the controller activities, for the functions and actions that need to take place within the rotation. These action include (among others) computing the phase (time relative to the TDC) of the injection and the quantity of fuel to be injected, but also checking whether the combustion occurred properly. Depending on the structure of the engine control application, these functions are implemented in tasks that are activated at each TDC, that is twice every crankshaft rotation (pseudo-cycle) or even more frequently (half-TDC).

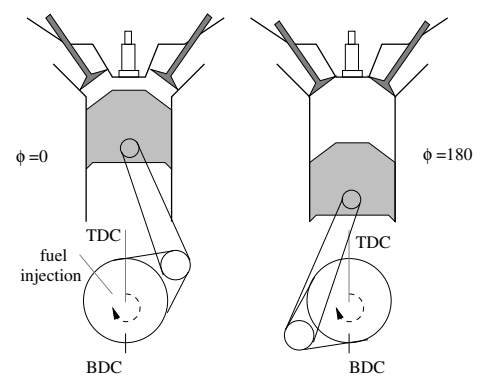

Figure 1: Relationship among engine phases and reference points in the crankshaft rotation period.

The problem with this type of tasks is that the time between two activations (at the TDC) is not constant, nor arbitrary, but depends on the rotation speed of the engine, which can vary 
within given ranges with a certain maximum acceleration. Thus, the acceleration bounds define a space of possible activation times which is not easy to capture and analyze without incurring in excessive pessimism (as it would, for example, if using the sporadic model).

To further complicate the treatment, the (worst-case) execution time of the functions executed by such tasks is typically not constant. At low revolution rates, the time interval between two reference points (the TDC for a set of cylinders) is large and allows the execution of sophisticated controls (and possibly multiple fuel injections). The same algorithm cannot be executed at higher revolution rates, because it would lead to an overload, generating several deadline misses. The typical approach adopted by the automotive industry [6] is to select the functions to be executed depending on the rotation speed. For most cars, the rotation speed typically varies between 600 and 6000 revolutions per minute (rpm), which maps to activation intervals between 100 and $10 \mathrm{~ms}$, for a complete revolution. A simplified implementation of such an adaptive behavior is shown in Figure 2.

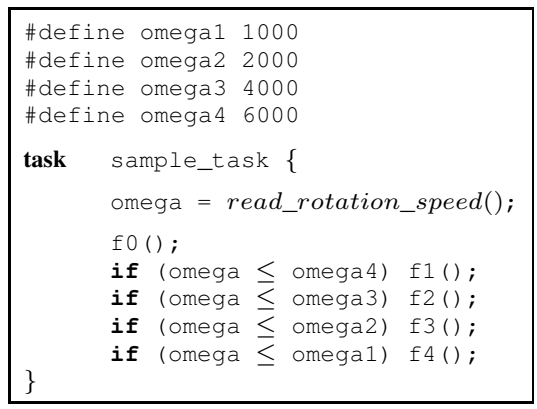

Figure 2: Typical implementation of a task with a functionality variable with the rotation speed of the crankshaft.

In this paper, the model proposed to describe such a type of engine control tasks is referred to as Adaptive Variable Rate task model, or AVR-model. Overall, a subset of the system tasks can be characterized as AVR tasks, typically executing together with classical periodic tasks under the control of a fixed-priority scheduler, as established in the automotive AUTOSAR standard.

Adaptivity of execution times can also be captured by considering that tasks may exhibit different execution modes. Note however, that mode change analysis [17], [18], [20] is not suited for analyzing AVR tasks, since their activation period is changing continuously, thus an infinite number of modes would be required to describe all possible situations.

\subsection{Related work}

In the real-time community, the problem related to AVR tasks was first presented by Buttle [6], as a common practice adopted in automotive applications to adapt the functionality and the computational requirements of engine control tasks for different rotation speeds.

A suitable model for AVR tasks with activation rates and execution times depending on the angular velocity of the engine has been proposed for the first time by Kim, Lakshmanan, and Rajkumar [12], who derived preliminary schedulability results under very simple assumptions. In particular, their analysis applies to a single AVR task with a period always smaller than the periods of the other tasks, and running at the highest priority level. In addition, all relative deadlines are assumed to be equal to periods and priorities are assigned based on the rate-monotonic algorithm.

Pollex et al. [16] also presented a sufficient schedulability analysis under fixed priorities, but they assumed that all the tasks with a variable rate depend on the same angular velocity, which can be arbitrary, but fixed. Moreover, the analysis is formulated using continuous intervals, hence it cannot be immediately translated into a practical schedulability test, whose complexity has not been evaluated.

The schedulability analysis of a generic set of AVR tasks under steady-state and dynamic conditions (considering a maximum acceleration of the engine) has been addressed by Buttazzo, Bini, and Buttle [4] under Earliest Deadline First (EDF) scheduling. They also provided a design method that allows computing the set of switching rotation speeds that keep the overall utilization below a desired bound.

The dynamic analysis of AVR tasks under fixed-priority scheduling has been considered by Davis et al. [7]. After discussing the complexity of the problem, they presented a sufficient test based on an Integer Linear Programming (ILP) formulation. Besides being only sufficient, their approach is based on a quantization of the instantaneous crankshaft rotation speed, which may introduce additional pessimism in the analysis to guarantee the safety of the test.

\subsection{Contributions and structure}

This paper provides the following novel contributions:

1) it presents an exact analysis of the worst-case interference generated by an AVR task in dynamic situations, under fixed priority systems and arbitrary deadlines, assuming realistic acceleration bounds derived from the automotive industry;

2) it discusses an efficient method for reducing the worst-case complexity of the exact analysis by identifying a set of cases that dominate the others, thus avoiding the need of a quantization of the engine state variable;

3) it presents a set of simulation experiments to compare the proposed analysis with the ILP-based test proposed by Davis et al. [7], showing that the interference computed by the ILP-based approach is always greater or equal to the one computed by our method.

The rest of the paper is organized as follows: Section 2 introduces the system model; Section 3 defines the interference of an AVR task and presents a brute force approach to compute it; Section 4 illustrates an efficient method for computing the exact interference by taking advantage of a pruning rule; Section 7 evaluates the performance of the proposed approach against the ILP-based test proposed by Davis et al. [7]; Section 8 states our conclusion and future work. 


\section{System model and notation}

This section presents the model for the task set and for the engine dynamics.

\subsection{Task model}

This paper considers a computing system running a set of $N$ preemptive real-time tasks $\Gamma=\left\{\tau_{1}, \tau_{2}, \ldots, \tau_{N}\right\}$ under fixed priorities and constrained deadlines. Each task $\tau_{i}$ generates an infinite sequence of jobs, $J_{i, 1}, J_{i, 2}, \ldots$ and can be either a periodic task, characterized by a fixed worst-case execution time (WCET) $C_{i}$, period $T_{i}$, and relative deadline $D_{i}$, or an AVR task, where all three of $C_{i}, T_{i}$, and $D_{i}$ are variable. For the sake of clarity, whenever needed, a rate-adaptive task may also be denoted as $\tau_{i}^{*}$.

The peculiarity of an AVR task $\tau_{i}^{*}$ is that its activation pattern and functionality are determined by the physical evolution of the engine. In particular, a generic job $J_{i, k}$ of an AVR task is activated when the crankshaft reaches predefined angular positions, thus the interarrival time between $J_{i, k}$ and $J_{i, k+1}$, denoted as period $T_{i, k}$, is a function of the crankshaft rotation speed. The sequence of activation times of the jobs of $\tau_{i}$ is denoted as $t_{i, 1}, t_{i, 2}, \ldots$, and we assume that activations are triggered at angular intervals of $\Delta \theta_{i}$. The relative deadline $D_{i, k}$ of job $J_{i, k}$ can be set as a non-decreasing function of $T_{i, k}$, with $D_{i, k} \leq T_{i, k}$.

The engine speed at time $t$ is denoted as $\omega(t)$ and, as a notation shortcut, the instantaneous speed at the activation time of the generic $k$-th job is indicated by $\omega_{k}=\omega\left(t_{k}\right)$. The speed is bounded in the interval $\left[\omega_{\min }, \omega_{\max }\right]$, where the minimum speed $\omega_{\min }$ defines the longest task period $T^{\max }=\Delta \theta / \omega_{\min }$, while the maximum speed $\omega_{\max }$ defines the smallest period $T^{\min }=$ $\Delta \theta / \omega_{\max }$ related to the first mode. The engine acceleration at time $t$, denoted by $\alpha(t)$, is assumed to be bounded between a maximum deceleration $\alpha^{-}$and a maximum acceleration $\alpha^{+}$, so that $\alpha(t) \in\left[\alpha^{-}, \alpha^{+}\right]$.

An AVR task $\tau_{i}^{*}$ is typically implemented as a set $\mathcal{M}_{i}$ of $M_{i}$ different modes

$$
\mathcal{M}_{i}=\left\{\left(C_{i}^{m}, \omega_{i}^{m}\right), m=1,2, \ldots, M_{i}\right\},
$$

each characterized by a certain functionality and WCET, kept constant when job activation speeds fall in the range $\left(\omega_{i}^{m}, \omega_{i}^{m+1}\right]$, where $\omega_{i}^{M_{i}+1}=\omega^{\text {min }}$ and $\omega_{i}^{1}=\omega_{\max }$ represent respectively the minimum and the maximum speed allowed by the system. Hence, the computation time of a generic AVR job $J_{i, k}$ can be expressed as a non-increasing step function $\mathcal{C}$ of the instantaneous speed $\omega_{i, k}$ at its release, that is,

$$
C_{i, k}=\mathcal{C}\left(\omega_{i, k}\right) \in\left\{C_{i}^{1}, \ldots, C_{i}^{M_{i}}\right\} .
$$

An example of $\mathcal{C}$ function is illustrated in Figure 3.

In the following sections, we are interested in computing the contribution to the interference of each individual AVR task (for periodic tasks the computation is trivial). To simplify the notation, the task index will be omitted from the task parameters whenever we refer to a single AVR task.

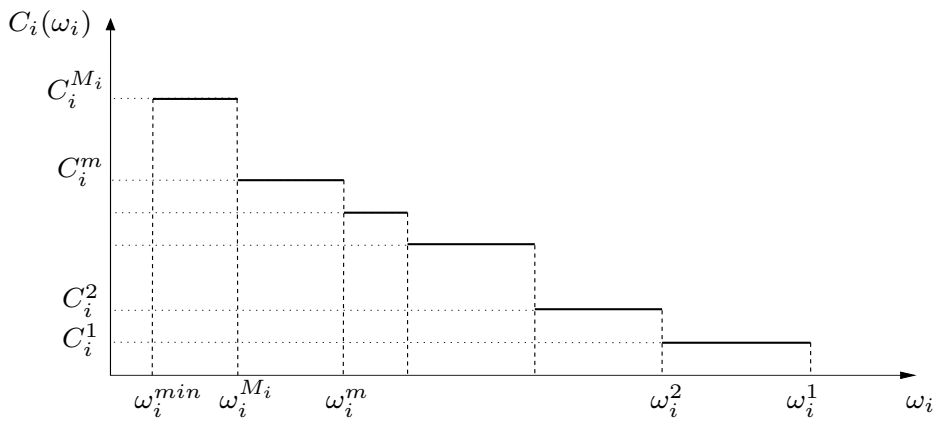

Figure 3: Computation time of an AVR task as a function of the speed.

\subsection{Engine dynamics and task activations}

To perform schedulability analysis in the presence of AVR tasks, it is important to characterize the relation between the engine dynamics and the task parameters. Given the current engine state $\left(\omega_{k}, \alpha_{k}\right)$ at time $t_{k}$, the time to the next job release is modeled (assuming a constant acceleration $\alpha_{k}$ ) as [4]:

$$
T\left(\omega_{k}, \alpha_{k}\right)=\frac{\sqrt{\omega_{k}^{2}+2 \alpha_{k} \Delta \theta}-\omega_{k}}{\alpha_{k}},
$$

where $\Delta \theta$ is the angular displacement that determines two consecutive job activations. Similarly, the instantaneous engine speed at the next job release is modeled (under the same assumption of constant acceleration) as $\Omega\left(\omega_{k}, \alpha_{k}\right)=\omega_{k}+\alpha_{k} T\left(\omega_{k}, \alpha_{k}\right)$, which gives:

$$
\Omega\left(\omega_{k}, \alpha_{k}\right)=\sqrt{\omega_{k}^{2}+2 \alpha_{k} \Delta \theta} .
$$

Given a job $J_{i, k}$ released with instantaneous speed $\omega_{k}$, the minimum interarrival time $\widetilde{T}^{m}\left(\omega_{k}\right)$ to have the next job $J_{i, k+1}$ released in mode $m$ (if reachable with the acceleration bounds), can be computed by Equation (2), substituting $\alpha_{k}$ obtained by Equation (3), which gives:

$$
\widetilde{T}^{m}\left(\omega_{k}\right)=\frac{2 \Delta \theta}{\omega_{k}+\omega^{m}} .
$$

Similarly, given a job $J_{i, k}$ released with instantaneous speed $\omega_{k}$ having period $T$, we define $\widetilde{\Omega}\left(\omega_{k}, T\right)$ as the instantaneous speed at the release of the next job $J_{i, k+1}$, which from Equation (4) results to be:

$$
\widetilde{\Omega}\left(\omega_{k}, T\right)=\frac{2 \Delta \theta}{T}-\omega_{k} .
$$

In addition, we denote as $\Omega^{n}$ the engine speed after $n$ job releases following the $k^{t h}$ job, computed as $\Omega^{n}\left(\omega_{k}, \alpha_{k}\right)=$ $\Omega\left(\Omega^{n-1}\left(\omega_{k}, \alpha_{k}\right), \alpha_{k}\right)$, where $\Omega^{0}\left(\omega_{k}, \alpha_{k}\right)=\omega_{k}$.

Figure 4 illustrates a range of possible scenarios that may determine the next activation for different speeds and accelerations. In particular, the figure shows the simultaneous evolution of the system state in terms of angular distance and angular speed, and the task activation pattern depending on the system state. In the interval $\left[t_{k}, t_{k+1}\right]$, an angular distance of $\Delta \theta$ is covered, while the angular speed is decreasing. 
The angular speed $\omega_{k}$ at time $t_{k}$ determines the activation of a new job $J_{k}$ with computation time $C_{k}=C\left(\omega_{k}\right)$. The dashed lines in the figure represent the cones within which the angular distance and speed may vary, determined by the extreme values of acceleration $\alpha^{+}$and $\alpha^{-}$. The dashed upward arrows in the bottom part of the figure represent the earliest and the latest activation for the subsequent job $J_{k+1}$, depending on the value of the acceleration $\alpha^{+}$and $\alpha^{-}$, respectively.

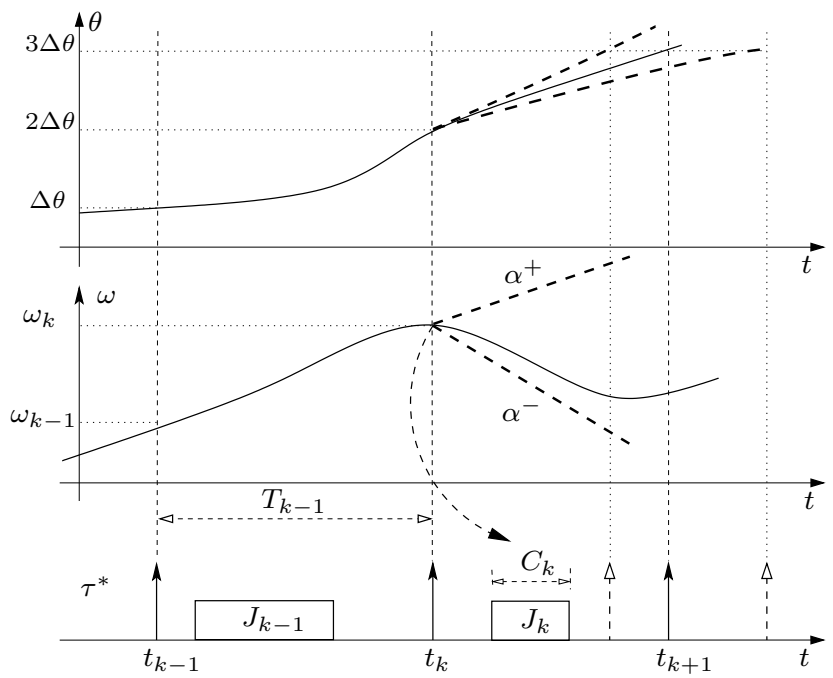

Figure 4: Dependency of task parameters from the system state.

\section{Characterizing the interference}

The interference caused by an AVR task $\tau_{h}$ on a lower priority task depends on the speed of the engine when the critical instant occurs. Hence, the interference is a function of the dynamics of the engine and should be computed for any initial speed $\omega_{0}$.

Let $I_{\omega_{0}}(t)$ denote the worst-case interference generated by an AVR task in the interval $[0, t]$, assuming that the critical instant occurs at time 0 , when the speed of the engine is $\omega_{0}$.

For each initial engine speed $\omega_{0}$, we determine $\Omega\left(\omega_{0}, \alpha\right)$ and $T\left(\omega_{0}, \alpha\right)$, considering a generic acceleration $\alpha$. A brute-force approach requires considering all possible values of $\alpha \in\left[\alpha^{-}, \alpha^{+}\right]$ to determine all possible subsequent job releases occurring in the interference window. Let us consider a job $J_{0}$ released at $t_{0}$, when the instantaneous speed is $\omega_{0}$. Such an activation gives rise to a family of possible instances of $J_{1}$, with period in the range $\left[T\left(\omega_{0}, \alpha^{+}\right), T\left(\omega_{0}, \alpha^{-}\right)\right]$and corresponding engine speed $\omega_{1}$ in the range $\left[\Omega\left(\omega_{0}, \alpha^{-}\right), \Omega\left(\omega_{0}, \alpha^{+}\right)\right]$, according to the model described in Section 2.

Similarly, the next activation gives rise to a set of possible job instances with period in $\left[T\left(\omega_{1}, \alpha^{+}\right), T\left(\omega_{1}, \alpha^{-}\right)\right]$and speed $\omega_{2} \in\left[\Omega\left(\omega_{1}, \alpha^{-}\right), \Omega\left(\omega_{1}, \alpha^{+}\right)\right]$. This reasoning applies recursively for each subsequent activation, until the end of the interference window, leading to a tree of possible job releases, as depicted in Figure 5.

Assuming the acceleration to vary continuously, the number of branches at each recursive step is theoretically infinite, but

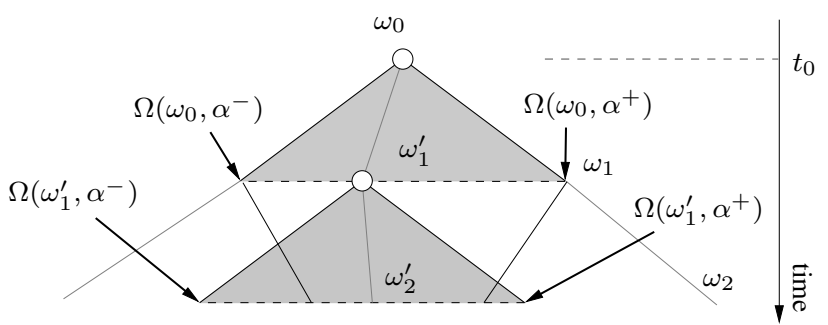

Figure 5: Tree of possible job releases of an AVR task.

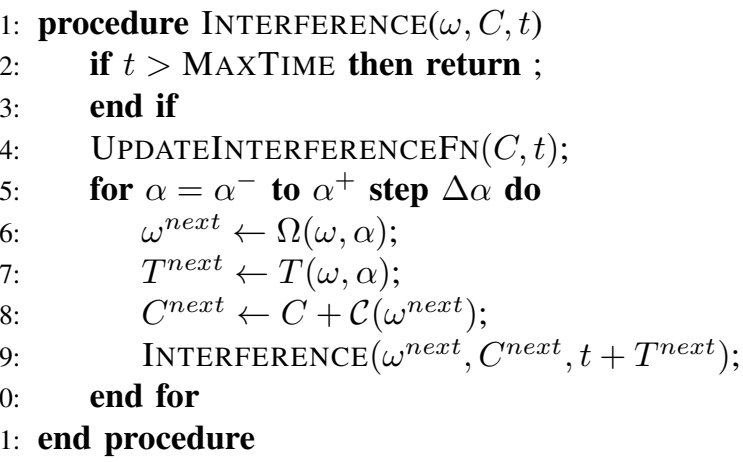

Figure 6: Procedure for computing the interference of an AVR performing an exhaustive tree-search.

can be bounded by making the acceleration range discrete with a given granularity. Of course, any quantization of the acceleration domain makes the analysis approximate, and a large granularity, while reducing the search complexity, increases the pessimism.

The pseudocode in Figure 6 summarizes the recursive procedure for computing the maximum interference following a bruteforce approach. The procedure is called by passing the initial engine speed $\omega_{0}$, the initial computation time $C_{0}=\mathcal{C}\left(\omega_{0}\right)$, and the current time instant.

At each recursive step, until the maximum deadline of any task (i.e. the largest length of the interference window that needs to be considered by the analysis, also denoted as MAXTIME, as in lines 2-3), we must:

- update the worst case interference function for a given value of $t$ (line 4);

- for each acceleration:

- compute the speed and the period related to the next job (lines 6-7);

- accumulate the overall computational request (line 8);

- recursively call the function INTERFERENCE (line 9).

The maximum interference function is a stepwise function storing the maximum interference time for each possible value $t$. The procedure UPDATEINTERFERENCEFN at line 4 simply saves the maximum cumulative value of the computational demand for the time instant passed as argument.

In summary, for a given $\omega_{0}$, the procedure generates a tree of job releases. Hence, computing the interference $I_{\omega_{0}}(t)$ is a search problem in the speed domain, and requires a complete visit 
of the tree. The interference $I_{\omega_{0}}(t)$ corresponds to the maximum among the interferences generated from all possible job sequences starting with speed $\omega_{0}$. This procedure is very expensive in terms of computational complexity, and intractable for most practical uses. The next section determines a pruning rule that cuts a significant number of branches, while still guaranteeing an exact interference analysis.

\section{Single-job interference}

To cut redundant branches in the search tree, we note that for each job release after the critical instant, only a finite set of critical job releases must be taken into account to derive the maximum interference. We explain how to compute such critical job releases, and then derive a pruning rule for the search problem presented in the previous section.

First of all, we construct the interference generated by an AVR task as the sum of the possible contributions of its individual jobs. To determine the potential interference generated by a single job $J_{a}$, it is necessary to consider all possible releases of $J_{a+1}$ compatible with the given acceleration $\alpha \in\left[\alpha^{-}, \alpha^{+}\right]$.

Definition 1: Given a job $J_{a}$ of an AVR task activated in mode $m$ at time $t_{a}$, the single-job interference $i_{\omega_{a}, m}(\delta)$ of $J_{a}$ is the maximum computational request generated by $J_{a}$ and $J_{a+1}$, in the interval $[0, \delta]$, for all possible releases of $J_{a+1}$ at $t_{a+1}=t_{a}+\delta$.

As explained in Section 3, job releases depend on the engine dynamics, and the future release times and modes of $J_{a+1}$ are constrained by the maximum/minimum acceleration of the engine. At time $t_{a}, i_{\omega_{a}, m}(0)=C^{m}$, since $J_{a}$ is released in mode $m$. Considering the maximum acceleration $\alpha^{+}$, it is easy to see that no job release can occur in the interval $0<\delta<T\left(\omega_{a}, \alpha^{+}\right)$; therefore, in this interval $i_{\omega_{a}, m}(\delta)=C^{m}$.

For $T\left(\omega_{a}, \alpha^{+}\right) \leq \delta \leq T\left(\omega_{a}, \alpha^{-}\right)$, an additional job release must be considered: the earliest considering the maximum acceleration $\alpha^{+}$, the latest considering the maximum deceleration $\alpha^{-}$. Depending on the engine dynamics, the released job can belong to a number of different modes. The larger the acceleration/deceleration range, the greater the number of possible modes. Since we are modeling the worst-case interference of an AVR task, we have to take into account all possible job releases for each possible mode $m^{\prime}$ with $\omega^{m^{\prime}} \in\left[\Omega\left(\omega_{a}, \alpha^{-}\right), \Omega\left(\omega_{a}, \alpha^{+}\right)\right]$. Finally, for $\delta>T\left(\omega_{a}, \alpha^{-}\right)$, no release of $J_{a+1}$ can occur; hence, $i_{\omega_{a}, m}(\delta)=\mathcal{C}\left(\Omega\left(\omega_{a}, \alpha^{-}\right)\right)$is the computational request of the latest possible job release time. Overall, $i_{\omega_{a}, m}(\delta)$ is a non-decreasing stepwise function, where each step represents the release of a different mode $m^{\prime}$.

Figure 7 shows an example of single-job interference assuming that the engine dynamics allows releasing jobs of two different modes in acceleration, and one in deceleration. As explained in Section 3, the interference $I_{\omega_{0}}(t)$ of an AVR task is the maximum sum of all its possible job requests. Therefore, it is possible to express $I_{\omega_{0}}(t)$ as the sum of time-shifted single job interferences.

The following theorem allows identifying the job instances for which the interference dominates the one for job instances at

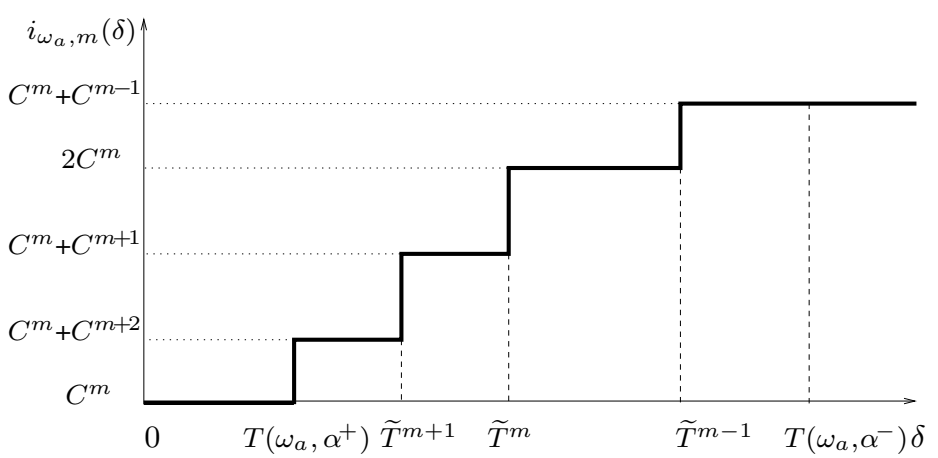

Figure 7: single job interference of a job activated at time $t_{a}$ in mode $m$.

other release times and therefore it can be used as a pruning rule to reduce the search space.

Theorem 1: Let $J_{a}$ and $J_{b}$ be two jobs released in mode $m$, and let $\omega_{a}$ and $\omega_{b}$ be the instantaneous engine speeds at their respective release times. If $\omega_{a} \geq \omega_{b}$ and $\mathcal{C}\left(\Omega\left(\omega_{a}, \alpha^{-}\right)\right)=$ $\mathcal{C}\left(\Omega\left(\omega_{b}, \alpha^{-}\right)\right)$, then $\forall \delta \geq 0 \quad i_{\omega_{a}, m}(\delta) \geq i_{\omega_{b}, m}(\delta)$.

Proof: The proof is trivial for $\omega_{a}=\omega_{b}$, therefore in the following we assume $\omega_{a}>\omega_{b}$. Since, for a given $\alpha, T\left(\omega, \alpha^{+}\right)$ and $T\left(\omega, \alpha^{-}\right)$are both monotonic decreasing functions in $\omega$, we have:

(i) $T\left(\omega_{a}, \alpha^{+}\right) \leq T\left(\omega_{b}, \alpha^{+}\right)$;

(ii) $T\left(\omega_{a}, \alpha^{-}\right) \leq T\left(\omega_{b}, \alpha^{-}\right)$.

From (i) we can derive that $i_{\omega_{a}, m}(\delta)=i_{\omega_{b}, m}(\delta)=C^{m}$ for $\delta<T\left(\omega_{a}, \alpha^{+}\right)$. For $T\left(\omega_{a}, \alpha^{+}\right) \leq \delta<T\left(\omega_{b}, \alpha^{+}\right)$we have $i_{\omega_{b}, m}(\delta)=C^{m}$ (job releases after $J_{b}$ cannot occur before $T\left(\omega_{b}, \alpha^{+}\right)$), while $i_{\omega_{a}, m}(\delta)$ can be larger because of the possible job releases following $J_{a}$. Hence, in the range $T\left(\omega_{a}, \alpha^{+}\right) \leq \delta<$ $T\left(\omega_{b}, \alpha^{+}\right)$, we have $i_{\omega_{a}, m}(\delta)>i_{\omega_{b}, m}(\delta)$.

For $\delta \geq T\left(\omega_{b}, \alpha^{+}\right)$two scenarios are possible:

- $T\left(\omega_{b}, \alpha^{+}\right) \leq T\left(\omega_{a}, \alpha^{-}\right)$, i.e., the two single-job interferences are overlapped in time. In this case, for $T\left(\omega_{b}, \alpha^{+}\right) \leq$ $\delta \leq T\left(\omega_{a}, \alpha^{-}\right)$, we have $\widetilde{\Omega}\left(\omega_{a}, \delta\right)<\widetilde{\Omega}\left(\omega_{b}, \delta\right)$, then $i_{\omega_{a}, m}(\delta) \geq i_{\omega_{b}, m}(\delta)$. In fact, fixing a generic time instant $\delta$ in this range, the single-job interference of $J_{a}$ is considering a lower instantaneous speed than the one of $J_{b}$, therefore it is modeling a greater or equal computational demand. In other words, since $\mathcal{C}(\omega)$ is non-increasing, then $\mathcal{C}\left(\widetilde{\Omega}\left(\omega_{a}, \delta\right)\right)>\mathcal{C}\left(\widetilde{\Omega}\left(\omega_{b}, \delta\right)\right)$

- $T\left(\omega_{b}, \alpha^{+}\right)>T\left(\omega_{a}, \alpha^{-}\right)$, i.e., the two single-job interferences are non-overlapped in time. In this case, for $T\left(\omega_{b}, \alpha^{+}\right) \leq \delta \leq T\left(\omega_{a}, \alpha^{-}\right)$, we have $i_{\omega_{a}, m}(\delta) \geq i_{\omega_{b}, m}(\delta)$ since $i_{\omega_{b}, m}(\delta)=C^{m}$.

In both cases, for $\delta>T\left(\omega_{a}, \alpha^{-}\right)$, we have $i_{\omega_{a}, m}(\delta)=i_{\omega_{b}, m}(\delta)$. This follows from (ii) and the hypothesis $\mathcal{C}\left(\Omega\left(\omega_{a}, \alpha^{-}\right)\right)=\mathcal{C}\left(\Omega\left(\omega_{b}, \alpha^{-}\right)\right)$, since $i_{\omega, m}(\delta)$ is non-decreasing.

Having shown that $i_{\omega_{a}, m}(\delta) \geq i_{\omega_{b}, m}(\delta)$ in each possible time interval, the theorem follows.

Figure 8 shows a typical scenario in which Theorem 1 holds, related to the case $T\left(\omega_{b}, \alpha^{+}\right) \leq T\left(\omega_{a}, \alpha^{-}\right)$. 


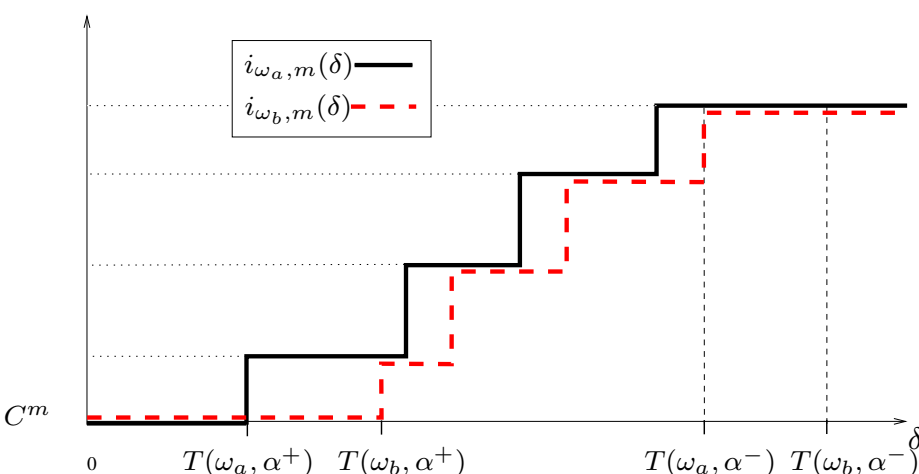

Figure 8: Example of a scenario for applying Theorem 1.

\section{Computing the exact interference}

In this section, Theorem 1 is used to build a pruning rule for reducing the search space. The complexity is reduced by finding a subset of potential job interferences that contribute to the exact interference $I_{\omega_{0}}(t)$.

Pruning rule properties. Consider a generic job $J_{i-1}$, and its immediate follower $J_{i}$. The release time of $J_{i}$ is variable and depends on the engine acceleration. Let $J_{i,(t)}$ be the instance of $J_{i}$ released at time $t$ ( $t$ is one of the activation times allowed by the acceleration range of the engine). The objective of the pruning rule is to identify a limited set $\mathcal{P}$ of critical job instances satisfying the following properties:

(i) for each $J_{i,(s)} \notin \mathcal{P}$, there must exist a $J_{i,(t)} \in \mathcal{P}$ for which the single job interference of $J_{i,(t)}$ dominates the one of $J_{i,(s)}$;

(ii) for each $J_{i,(s)} \notin \mathcal{P}$, there must exist a $J_{i,(t)} \in \mathcal{P}$ such that the interference generated by all job releases following $J_{i,(s)}$ is dominated by the interference of at least one set of possible job instances following $J_{i,(t)}$.

Property (i) allows to eliminate all the job instances $J_{i,(s)} \notin \mathcal{P}$, while property (ii) allows to discard the entire sub-tree of job instances released after $J_{i,(s)}$.

\subsection{Job instances for property (i)}

Suppose that the critical instant occurs when the instantaneous engine speed is $\omega_{0}$ and the AVR task is in mode $m$. The single job interference $i_{\omega_{0}, m}(t)$ is the initial value of $I_{\omega_{0}}(t)$. Using Theorem 1 , it is possible to identify a set $\mathcal{P}^{(i)} \subset \mathcal{P}$ of job releases for which their single job interference dominates the others.

Theorem 1 only applies to jobs activated in the same mode. Hence, the set $\mathcal{P}^{(i)}$ includes at least one job instance for each mode $m$ such that $\widetilde{T}^{m}\left(\omega_{0}\right) \in\left[T\left(\omega_{0}, \alpha^{+}\right), T\left(\omega_{0}, \alpha^{-}\right)\right]$. For each mode, we need to consider the earliest job release, in order to satisfy the theorem hypothesis $\omega_{a} \geq \omega_{b}$. Formally, we refer to such job releases as the earliest distinct mode changes (EDMCs). EDMCs can also be defined as the set of the job instances $J_{i,(t)}$ for which their current period $T_{i}$ belongs to the set $T_{\text {EDMC }}$, which includes the period corresponding to the earliest possible arrival of a job instance ( $\max$ acceleration) and all the periods that correspond to mode changes, that is

$$
\begin{aligned}
T_{\mathrm{EDMC}}= & \left\{T\left(\omega_{0}, \alpha^{+}\right)\right\} \cup\left\{T \mid T \in\left[T\left(\omega_{0}, \alpha^{+}\right), T\left(\omega_{0}, \alpha^{-}\right)\right]\right. \\
& \text {such that } \left.T=\widetilde{T}^{m}\left(\omega_{0}\right) \text { for some } m \in 1, \ldots, M\right\} .
\end{aligned}
$$

In the example of Figure 7 , the set $T_{\text {EDMC }}$ is given by the time instants on the $\mathrm{x}$-axis corresponding to the steps of $i_{\omega_{0}, m}(t)$.

However, the set $\mathcal{P}^{(i)}$ needs to include other job instances besides those in EDMCs, since there can be job instances $J_{i,(q)}$ that are not dominated by any of the instances in EDMC. This can happen because the instances in EDMC do not necessarily guarantee the last hypothesis of Theorem 1. For these job releases $J_{i,(q)}$, there is no $J_{i,(t)}$ having period $T_{i,(t)} \in T_{\text {EDMC }}$ with $\mathcal{C}\left(\Omega\left(\omega_{i,(t)}, \alpha^{-}\right)\right)=\mathcal{C}\left(\Omega\left(\omega_{i,(q)}, \alpha^{-}\right)\right)$.

The set EDMC needs to be extended to include other instances. Hence, the set EDMC $=\left\{J_{i,\left(t_{1}\right)}, J_{i,\left(t_{2}\right)}, \ldots J_{i,\left(t_{z}\right)}\right\}$ is sorted by increasing arrival times. $J_{i,\left(t_{1}\right)}$ is the earliest possible instance. Then, for every interval $\left[J_{i,\left(t_{m}\right)}, J_{i,\left(t_{m+1}\right)}\right]$ we need to look for an intermediate time point (arrival time) $t_{q}$ that corresponds to an instance not dominated by the endpoint $J_{i,\left(t_{m}\right)}$ because $\mathcal{C}\left(\Omega\left(\omega_{\left.i, t_{m}\right)}, \alpha^{-}\right)\right) \neq \mathcal{C}\left(\Omega\left(\omega_{\left.i, t_{q}\right)}, \alpha^{-}\right)\right)$.

The candidate time instants $t_{q}$ can be computed by considering that they can only belong to the set of points for which $\Omega\left(\omega_{\left.i, t_{q}\right)}, \alpha^{-}\right)=\omega^{m}$ for one of the modes $m$.

If such $t_{q}$ exists, it is added to the set and the test continues in the interval $\left[J_{i,\left(t_{q}\right)}, J_{i,\left(t_{m+1}\right)}\right]$. When all points in $\left[J_{i,\left(t_{m}\right)}, J_{i,\left(t_{m+1}\right)}\right]$ are checked, the algorithm moves to the next time interval in the original set EDMC until all the dominant job instances are found.

\subsection{Job instances for property (ii)}

Unfortunately, Theorem 1 is not sufficient to discard all the single-job interferences generated by the jobs following $J_{i,(s)}$. For example, as illustrated in Figure 9, consider a generic job instance $J_{i,(s)}$ for which the single job interference is dominated by $J_{i,(t)} \in \mathcal{P}^{(i)}$. Since by hypothesis we have $\omega_{i,(s)} \leq \omega_{i,(t)}$, a job $J_{i+1,\left(s^{\prime}\right)}$ immediately following $J_{i,(s)}$ could have a lower speed than all the possible jobs instances $J_{i+1,\left(t^{\prime}\right)}$ immediately following $J_{i,(t)}$. Formally, the maximum speeds for $J_{i+1,\left(t^{\prime}\right)}$ and $J_{i+1,\left(s^{\prime}\right)}$ are respectively $\omega_{i+1,\left(t^{\prime}\right)}=\Omega\left(\Omega\left(\omega_{i,(t)}, \alpha^{-}\right), \alpha^{-}\right)$and $\omega_{i+1,\left(s^{\prime}\right)}=\Omega\left(\Omega\left(\omega_{i,(s)}, \alpha^{-}\right), \alpha^{-}\right)<\omega_{i+1,\left(t^{\prime}\right)}$. Hence, $J_{i,(s)}$ cannot be pruned, since it could be that $\mathcal{C}\left(\omega_{i+1,\left(s^{\prime}\right)}\right)>\mathcal{C}\left(\omega_{i+1,\left(t^{\prime}\right)}\right)$.

The following theorem addresses this issue. To compact the readability of the theorem, we define the following set:

$$
\overline{\mathbb{N}}=\left\{k \in \mathbb{N}^{+} \mid \min \left(\Omega^{k}\left(\omega_{a}, \alpha^{-}\right), \Omega^{k}\left(\omega_{b}, \alpha^{-}\right)\right) \leq \omega^{\min }\right\} .
$$

Theorem 2: Let $J_{a}$ and $J_{b}$ be two jobs released in mode $m$, and let $\omega_{a}$ and $\omega_{b}$ be the instantaneous engine speeds at their respective release times. If $\omega_{a} \geq \omega_{b}$ and $\forall n \in \overline{\mathbb{N}}$ $\mathcal{C}\left(\Omega^{n}\left(\omega_{a}, \alpha^{-}\right)\right)=\mathcal{C}\left(\Omega^{n}\left(\omega_{b}, \alpha^{-}\right)\right)$, then $\forall t \geq 0 I_{\omega_{a}}(t) \geq I_{\omega_{b}}(t)$.

Proof: The proof is trivial for $\omega_{a}=\omega_{b}$, therefore in the following we assume $\omega_{a}>\omega_{b}$. We must show that, for each 


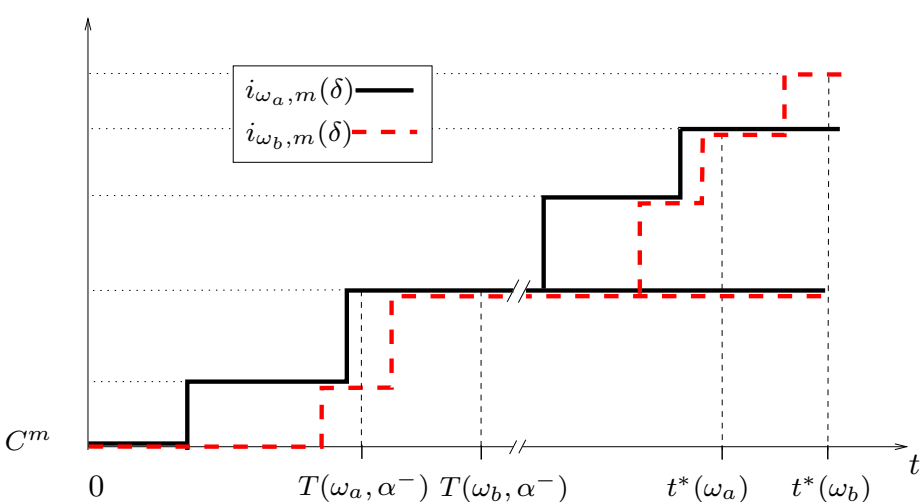

Figure 9: Example in which Theorem 1 is not sufficient to discard all the interferences generated by the jobs following $J_{b}$. The function $t^{*}(\omega)$ in the graph is defined as $t^{*}(\omega)=$ $T\left(\omega, \alpha^{-}\right)+T\left(\Omega\left(\omega, \alpha^{-}\right), \alpha^{-}\right)$.

possible job $J_{b+n}$ following $J_{b}$ there exists a job $J_{a+n}$ following $J_{a}$ such that the single job interference of $J_{b+1}$ is dominated by that of $J_{a+1}$. To do this, we apply Theorem 1 by induction.

Since $\Omega^{n}(\omega, \alpha)$ is a monotonic increasing function, we have

$$
\begin{aligned}
& \forall n \in \overline{\mathbb{N}} \quad \Omega^{n}\left(\omega_{a}, \alpha^{-}\right) \geq \Omega^{n}\left(\omega_{b}, \alpha^{-}\right) ; \\
& \forall n \in \overline{\mathbb{N}} \quad \Omega^{n}\left(\omega_{a}, \alpha^{+}\right) \geq \Omega^{n}\left(\omega_{b}, \alpha^{+}\right) .
\end{aligned}
$$

For each $n$, two scenarios are possible as shown in Figure 10: a) $\Omega^{n}\left(\omega_{a}, \alpha^{-}\right)>\Omega^{n}\left(\omega_{b}, \alpha^{+}\right)$; in this case, to apply Theorem 1 on each $J_{b+n}$, it is sufficient to take $J_{a+n}$ having $\omega_{a+n}=$ $\Omega^{n}\left(\omega_{a}, \alpha^{-}\right)$. Let then $\omega_{b+n} \in\left[\Omega^{n}\left(\omega_{b}, \alpha^{-}\right), \Omega^{n}\left(\omega_{b}, \alpha^{+}\right)\right]$. This choice makes the three hypotheses satisfied, since:

- $\omega_{a+n} \geq \omega_{b+n}$ follows from $\Omega^{n}\left(\omega_{a}, \alpha^{-}\right)>\Omega^{n}\left(\omega_{b}, \alpha^{+}\right)$;

- $\mathcal{C}\left(\Omega\left(\omega_{a+n}, \alpha^{-}\right)\right)=\mathcal{C}\left(\Omega\left(\omega_{b+n}, \alpha^{-}\right)\right)$follows from the hypothesis $\mathcal{C}\left(\Omega^{n}\left(\omega_{a}, \alpha^{-}\right)\right)=\mathcal{C}\left(\Omega^{n}\left(\omega_{b}, \alpha^{-}\right)\right) \forall n$. This can be shown by replacing the definition of the function $\Omega^{n+1}(\omega, \alpha)$ obtaining $\mathcal{C}\left(\Omega\left(\Omega^{n}\left(\omega_{a+n}, \alpha^{-}\right), \alpha^{-}\right)\right)=$ $\mathcal{C}\left(\Omega\left(\Omega^{n}\left(\omega_{b+n}, \alpha^{-}\right), \alpha^{-}\right)\right)$. Since $\mathcal{C}(\omega)$ is a monotonic non-decreasing function, the hypothesis is verified for all $\omega_{b+n}$.

- $J_{a+n}$ has the same mode as $J_{b+n}$ : again this is ensured by considering the hypothesis $\mathcal{C}\left(\Omega^{n}\left(\omega_{a}, \alpha^{-}\right)\right)=$ $\mathcal{C}\left(\Omega^{n}\left(\omega_{b}, \alpha^{-}\right)\right) \forall n$.

b) $\Omega^{n}\left(\omega_{a}, \alpha^{-}\right) \leq \Omega^{n}\left(\omega_{b}, \alpha^{+}\right)$; in this case, for each possible job $J_{b+n}$ having $\omega_{b+n} \in\left[\Omega^{n}\left(\omega_{a}, \alpha^{-}\right), \Omega^{n}\left(\omega_{b}, \alpha^{+}\right)\right]$there exists a job $J_{a+n}$ having $\omega_{a+n} \in\left[\Omega^{n}\left(\omega_{a}, \alpha^{-}\right), \Omega^{n}\left(\omega_{a}, \alpha^{+}\right)\right]$, with $\omega_{a+n}=\omega_{b+n}$. This result implies directly $\mathcal{C}\left(\Omega\left(\omega_{a+n}, \alpha^{-}\right)\right)=$ $\mathcal{C}\left(\Omega\left(\omega_{b+n}, \alpha^{-}\right)\right)$. Since in this interval also the periods for $J_{b+n}$ and $J_{a+n}$ are overlapped, each $J_{b+n}$ can be mapped to $J_{a+n}$ having the same mode. The application of Theorem 1 is then straightforward.

On the other hand, for each possible job $J_{b+n}$ having $\omega_{b+n} \in$ $\left[\Omega^{n}\left(\omega_{b}, \alpha^{-}\right), \Omega^{n}\left(\omega_{a}, \alpha^{-}\right)\right]$, the same considerations made for the upper case hold. Hence, it is sufficient to take $J_{a+n}$ having $\omega_{a+n}=\Omega^{n}\left(\omega_{a}, \alpha^{-}\right)$.

Hence the theorem follows. a)

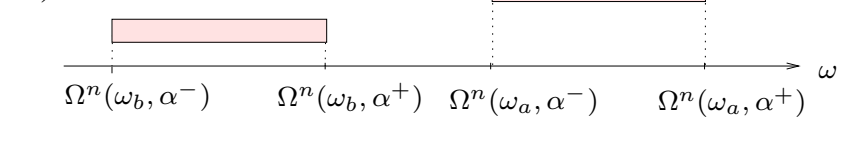

b)

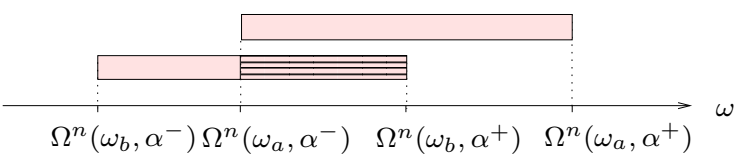

Figure 10: Possible scenarios for Theorem 2.

By using Theorem 2, it is possible to obtain the complete set $\mathcal{P}$ required by the pruning rule. To identify critical job releases belonging to $\mathcal{P}$ we proceed in the same way as for $\mathcal{P}^{(i)}$ : the only difference is that Theorem 2 is applied in place of Theorem 1 .

\subsection{Dominant initial velocities}

Theorem 2 can also be exploited to find a reduced set of initial instantaneous speeds that must be considered for the interference computation. Let $\omega_{a}$ and $\omega_{b}$ be two instantaneous speeds at which the critical instant may occur. If the hypotheses of Theorem 2 are verified, then we can conclude that $\forall t I_{\omega_{a}}(t) \geq I_{\omega_{b}}(t)$, that is, the interference for $\omega_{b}$ is entirely dominated by the one for $\omega_{a}$.

It follows that, given a range of possible engine speeds, we can compute a set of dominant speeds in this range in the very same way as we identified critical job releases applying the pruning rule in the range of speeds determined by $\alpha^{-}$and $\alpha^{+}$.

In the following,

- $\Lambda_{i}\left(\omega_{1}, \omega_{2}\right)$ denotes the set of dominant speeds for an AVR task $\tau_{i}^{*}$ when the engine speed can vary in the range $\left(\omega_{1}, \omega_{2}\right]$.

- $\Lambda_{i}^{*}=\Lambda_{i}\left(\omega_{i}^{\min }, \omega_{i}^{\max }\right)$ denotes the set of dominant speeds of task $\tau_{i}^{*}$ for all possible speeds.

- $\Lambda_{i}^{m}$ denotes the set of dominant speeds of task $\tau_{i}^{*}$ when the engine speed can vary in the range compatible with mode $m$; that is,

$$
\Lambda_{i}^{m}=\Lambda_{i}\left(\omega^{m}, \omega^{m+1}\right) .
$$

This is a key point to simplify the analysis with respect to the brute-force approach described in Section 3, and also to the ILP formulation proposed by Davis et al. [7], since both approaches require to consider the complete range of possible initial speeds.

\section{Tight schedulability analysis}

In this section we show how to use the interference of an AVR task $\tau_{H}^{*}\left(I_{H, \omega_{0}}\right)$ to perform a response time analysis [1] of a generic task set consisting of AVR and periodic/sporadic tasks.

The proposed analysis is based on the interference $I_{\omega}(t)$, which is the envelope of all the possible sequences of singlejob interferences in $[0, t]$. As shown by Stigge and Yi [19], the use of an interference envelope in response-time analysis can lead to an over-approximation of the exact response time, hence the proposed schedulability tests are only sufficient. 
To better explain this fact, consider the example shown in Figure 11, where $i_{a}(t)$ and $i_{b}(t)$ are two time-shifted singlejob interferences (remember that $I_{\omega}(t)$ can be expressed as the sum of time-shifted single job interferences). As shown in the figure, the interference envelope $I_{\omega}(t)$ leads to a response time $R$ (corresponding to the first point in which $I_{\omega}(t)$ matches the processor supply). Please note that the response time $R$ is originated by interference values of $i_{a}(t)$; however, the schedule represented by $i_{a}(t)$ has response time $R_{a}<R$. In other words, the response time $R$ is over-approximated since the schedule that originate it has experienced an idle-time before $t=R$. Overall, we can conclude that the use of an interference envelope allows deriving safe upper-bounds on the response time, but introduces pessimism by losing information related to each single schedule potentially contributing to the envelope.

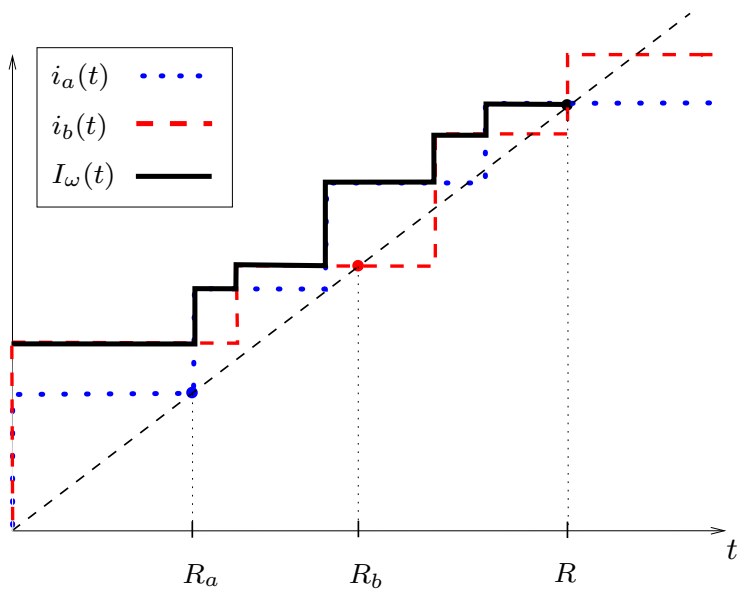

Figure 11: Example of a scenario in which the use of an interference envelope leads to an over-approximated response time.

We consider two cases to compute:

- the interference of an AVR task $\tau_{H}^{*}$ on a periodic/sporadic task $\tau_{L}$ with lower priority;

- the interference of an AVR task $\tau_{H}^{*}$ on another AVR task $\tau_{L}^{*}$ with lower priority.

When computing the interference of $\tau_{H}^{*}$ on a periodic/sporadic task or an AVR task with an independent source of activation events (independent $\omega_{0}$ ), the worst-case combination for any initial speed of $\tau_{H}^{*}$ needs to be considered. To do so, the envelope $I_{H}(t)$ has to be computed as the maximum among the interference functions $I_{H, \omega_{0}}(t)$ for each initial speed. Using Theorem 2, it is sufficient to consider only the dominant speeds in $\Lambda_{H}^{*}$, hence

$$
I_{H}(t)=\max _{\omega_{0} \in \Lambda_{H}^{*}}\left\{I_{H, \omega_{0}}(t)\right\} .
$$

To simplify the notation of the test, we also define the (finite) set $\mathcal{I}_{H}(t)$ of time points less than or equal to the argument $t$, in which the step function $I_{H}()$ changes its value.

\subsection{AVR Interference on a periodic task}

To analyze the interference of an AVR task $\tau_{H}^{*}$ on a lower priority periodic/sporadic task $\tau_{L}$, every possible initial speed $\omega_{0}$ of $\tau_{H}^{*}$ has to be considered at the beginning of the critical instant of $\tau_{L}$. Therefore, the feasibility condition for $\tau_{L}$ becomes:

$$
\forall \omega_{0} \quad \exists t \leq D_{L}: C_{L}+I_{H, \omega_{0}}(t) \leq t,
$$

which is the same as

$$
\exists t \leq D_{L}: C_{L}+\max _{\omega_{0}}\left\{I_{H, \omega_{0}}(t)\right\} \leq t .
$$

which, using Theorem 2 and Equation (6), reduces to

$$
\exists t \leq D_{L}: C_{L}+I_{H}(t) \leq t .
$$

\subsection{AVR Interference on an AVR task}

When analyzing the interference of an AVR-task $\tau_{H}^{*}$ over another lower priority AVR task $\tau_{L}^{*}$, two cases can be distinguished. Let $\omega_{H}$ and $\omega_{L}$ be the variables describing the speeds of the activation sources for $\tau_{H}^{*}$ and $\tau_{L}^{*}$, respectively. As a first case we consider the two speeds to be independent, while in the second case we consider them related.

When rotation speeds are independent, the low-priority task $\tau_{L}^{*}$ is schedulable if

$$
\forall \omega_{H} \omega_{L} \quad \exists t \leq D_{L}\left(\omega_{L}\right): \mathcal{C}_{L}\left(\omega_{L}\right)+I_{H, \omega_{H}}(t) \leq t .
$$

Since the WCET of $\tau_{L}^{*}$ depends on its execution mode, Equation (8) must be true for all modes. In addition, the full set of speeds of $\tau_{H}^{*}$ can be considered using Equation (6), thus $\tau_{L}^{*}$ is feasible if $\forall m=1, \ldots, M_{L}$ and $\forall \omega_{L} \in\left(\omega_{L}^{m+1}, \omega_{L}^{m}\right]$

$$
\exists t \leq D_{L}\left(\omega_{L}\right): C_{L}^{m}+I_{H}(t) \leq t .
$$

The previous formula should be evaluated for the full set of deadlines for mode $m$, denoted as $\mathcal{D}_{L}^{m}$ :

$$
\mathcal{D}_{L}^{m}=\left\{t \mid t \in\left(D_{L}\left(\omega_{L}^{m-1}\right), D_{L}\left(\omega_{L}^{m}\right)\right]\right\} .
$$

Note however, that we do not need to consider an infinite number of points in $\mathcal{D}_{L}^{m}$, since $I_{H}(t)$ only changes its value in the finite set of time points $\mathcal{I}_{H}\left(\max \left\{\mathcal{D}_{L}^{m}\right\}\right)$ (up to the maximum deadline for the mode). Therefore, $\tau_{L}^{*}$ can be feasibly scheduled if $\forall m=$ $1, \ldots, M_{L}$ and $\forall t \in \mathcal{D}_{L}^{m} \cap \mathcal{I}_{H}\left(\max \left\{\mathcal{D}_{L}^{m}\right\}\right)$

$$
C_{L}^{m}+I_{H}(t) \leq t
$$

When $\omega_{H}$ and $\omega_{L}$ are related to a common speed $\omega_{0}$, the previous analysis is pessimistic and needs to be refined. The response time analysis is a function of $\omega_{0}$ and $\tau_{L}^{*}$ can be feasibly scheduled if

$$
\forall \omega_{0} \quad \exists t \leq D_{L}\left(\omega_{0}\right): \mathcal{C}_{L}\left(\omega_{0}\right)+I_{H, \omega_{0}}(t) \leq t
$$

Considering each mode separately we have:

$$
\begin{aligned}
& \forall m=1, \ldots, M_{L} \quad \forall \omega_{0} \in\left(\omega_{L}^{m+1}, \omega_{L}^{m}\right] \\
& \exists t \leq D_{L}\left(\omega_{0}\right) \quad: \quad C_{L}^{m}+I_{H, \omega_{0}}(t) \leq t .
\end{aligned}
$$


To compute the interference of the high priority task, we consider the set of its dominant speeds within the speed range of every mode for $\tau_{L}^{*}$. The range of speeds for each mode is partitioned in the intervals defined by the dominant speeds of $\tau_{H}^{*}$. Let $\left(\omega^{d_{i-1}}, \omega^{d_{i}}\right]$ be the generic interval between two of such dominant speeds $\left(\omega^{d_{j}} \in \Lambda_{H}^{*}\right)$, and let $p_{m}$ be the number of such intervals for mode $m$. For each interval $\left(\omega^{d_{i-1}}, \omega^{d_{i}}\right]$, since the deadline is a non-increasing function of the speed, the shortest deadline for $\tau_{L}^{*}$ (replacing the term $D_{L}\left(\omega_{0}\right)$ in the formula) corresponds to the dominant $\omega^{d_{i}}$ at the highest end of the interval. $\omega^{d_{i}}$ also allows to upper bound the interference term $I_{H, \omega^{d_{i}}}\left(D_{L}\left(\omega^{d_{i}}\right)\right) \geq I_{H, \omega_{0}}\left(D_{L}\left(\omega_{0}\right)\right)$ for every $\omega_{0} \in\left(\omega^{d_{i-1}}, \omega^{d_{i}}\right]$. Hence, the schedulability of $\tau_{L}^{*}$ can only be tested in $p_{m}$ points:

$$
\begin{aligned}
& \forall m=1 \ldots M_{L}, \quad i=1 \ldots p_{m} \\
& \quad \exists t \leq D_{L}\left(\omega^{d_{i}}\right): C_{L}^{m}+I_{H, \omega^{d_{i}}}(t) \leq t .
\end{aligned}
$$

\section{Evaluation}

This section presents an evaluation of the proposed analysis method carried out on task data that are representative for an engine control system. The application was provided in the context of the INTERESTED EU project [9] and consists of a set of periodic and AVR tasks. One of these tasks is activated at the TDC mark and is characterized by 6 execution modes, reported in Table 1 , and by a maximum period $T_{\max }=120 \mathrm{~ms}$ (corresponding to $500 \mathrm{rpm}$ ).

\begin{tabular}{|l|c|c|c|c|c|c|}
\hline mode & 1 & 2 & 3 & 4 & 5 & 6 \\
\hline \hline$r p m$ & 6500 & 5500 & 4500 & 3500 & 2500 & 1500 \\
\hline$T^{m}(m s)$ & 9.23 & 10.91 & 13.33 & 17.15 & 24 & 40 \\
\hline$C^{m}(\mu \mathrm{s})$ & 246 & 277 & 343 & 424 & 576 & 965 \\
\hline
\end{tabular}

Table 1: Task parameters used in the evaluation.

Such a task is used to compare the accuracy and performance of the proposed approach with respect to the sufficient ILP-based method proposed by Davis et al. [7]. Note, however, that while in [7] the instantaneous speed is estimated as the average speed in the previous interarrival time, in our model the instantaneous speed is assumed to be known.

Before describing the results of the experiments, a few considerations are necessary to explain the terms of the comparison. The interference analysis is a function of two variables: the initial engine speed $\omega_{0}$ and the length $t$ of the interference window. The ILP method can only compute the interference for a set of discrete values of $t$ and $\omega_{0}$. Our analysis, thanks to the derivation of the dominant speeds, can actually compute the exact interference function for all the values $t$ and $\omega_{0}$ in their continuous ranges.

To run the experiments, the ILP method has been implemented on a CPLEX solver running on an 8-core Intel Xeon at $2.8 \mathrm{GHz}$, while our algorithm was implemented as MATLAB code on a dual-core laptop Intel i7 at $2.5 \mathrm{GHz}$. The run time of the two algorithms resulted in the order of few seconds to compute the maximum interference with the ILP algorithm, and about one minute to characterize the interference in the whole time interval with our algorithm.

Two experiments have been carried out. The first experiment is meant to compare the quality of the analysis in the domain of the initial speed $\omega_{0}$. Figure 12 plots the interference $I_{\omega_{0}}(t)$ generated by the AVR task in a time interval of $100 \mathrm{~ms}$ for a set of initial engine speeds $\omega_{0}$ between $1500 \mathrm{rpm}$ and $6500 \mathrm{rpm}$ and a maximum acceleration of $1.6210^{-4} \mathrm{rev} / \mathrm{msec}^{2}$. As suggested by Davis at al. [7], a quantization step of $100 \mathrm{rpm}$ was used to define the set of discrete values for $\omega_{0}$. As shown in the figure, our analysis is able to achieve an improvement of 20 percent, or higher, for specific speeds, exhibiting an average improvement of about 10 percent.

The ILP formulation proposed in [7] presents some inconsistencies when low initial speeds are considered, leading to an incorrect interference for low values of the initial speed $\omega_{0}$. Such a problem has been fixed by the authors in a technical report [8], which is taken as a reference for our comparison.

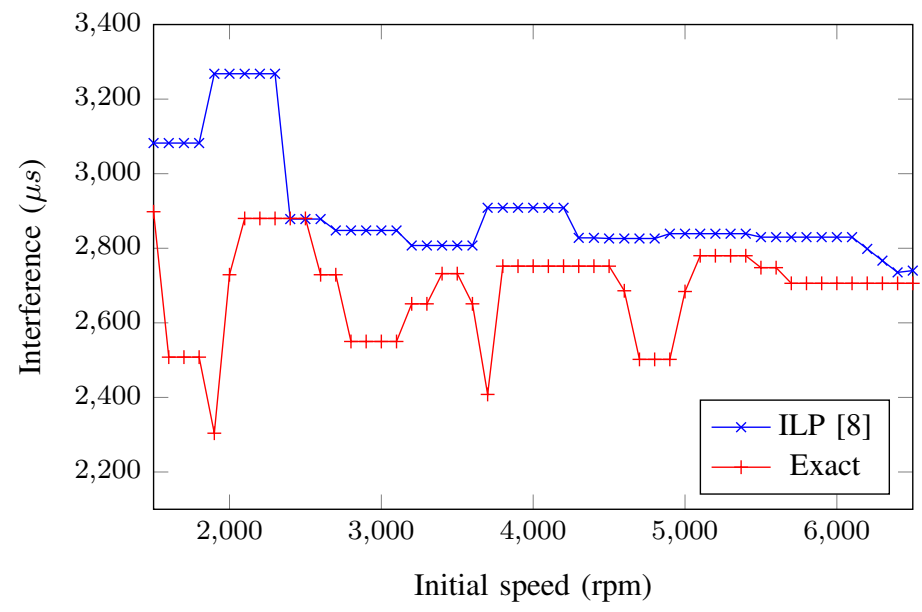

Figure 12: AVR Interference for different initial engine speeds, in a time interval of $100 \mathrm{~ms}$.

In a second experiment, we evaluated the pessimism of the ILP method in the dimension of time, for an initial engine speed $\omega_{0}=5600 \mathrm{rpm}$. Figure 13 illustrates the interference function computed by the two algorithms when the interference interval is varied from $30 \mathrm{~ms}$ to $75 \mathrm{~ms}$. In this time range, the pessimism of the ILP method remains of approximately 10 percent, and tends to increase for larger values of $t$.

\section{Conclusions}

This paper presented an exact analysis of the worst-case interference generated by an adaptive variable-rate task under fixedpriority scheduling and arbitrary deadlines. The work advances the state of the art, since previous papers on fixed-priority AVR tasks either focused on special simplified cases [12] or proposed an approximate ILP test [7] based on a quantization of the state variable (i.e., the crankshaft rotation speed in engine control applications) determining the task activation rate. 


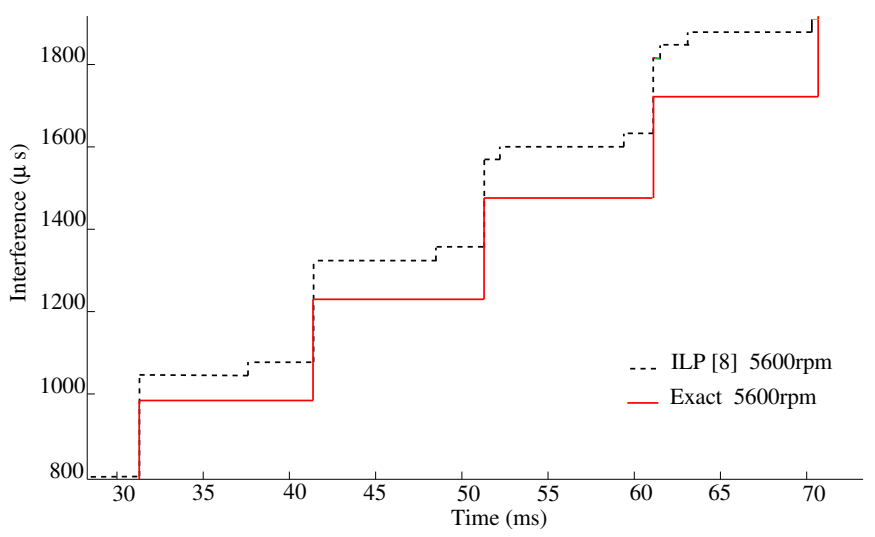

Figure 13: AVR Interference for different time intervals, with $\omega_{0}$ $=5600 \mathrm{rpm}$.

The second contribution of this work has been the identification of a finite subset of dominant speeds that determine the exact worst-case interference of an AVR task. Thanks to this result, the interference analysis does not need to be performed for all possible values of the engine speed, with a given quantization, but can be determined by considering only the set of dominant speeds.

It is worth observing that the set of dominant speeds is not only important for reducing the complexity of the search, but also because it allows getting rid of the quantization of the state variable, which can make the schedulability analysis unsafe. In fact, by quantizing the state variable, the schedulability test is performed only in a subset of all possible values, so there can be points (not considered in the test) in which the schedulability test is not satisfied.

The simulation results reported in Section 7 confirm that the interference computed by the ILP approximated method in the discrete values of the initial speed $\omega_{0}$ is always higher or equal to the interference computed by the approach proposed in this paper, in the same points.

As future work, we plan to extend the task model to consider more realistic design solutions considered in the automotive application domain, like AVR tasks with an initial phase and mode transitions with hysteresis.

\section{Acknowledgements}

The authors like to thank Robert I. Davis, Timo Feld, Victor Pollex, and Frank Slomka for the interesting and fruitful discussions that helped to improve this work.

\section{References}

[1] N. Audsley, A. Burns, M. Richardson, K. Tindell, and A. Wellings. Applying new scheduling theory to static priority pre-emptive scheduling. Software Engineering, 8(5):284-292, Sept. 1993.

[2] S. K. Baruah, D. Chen, S. Gorinsky, and A. K. Mok. Generalized multiframe tasks. Real-Time Systems, 17(1):5-22, July 1999.
[3] G. Buttazzo, L. Abeni, and G. Lipari. Elastic task model for adaptive rate control. In IEEE Real Time System Symposium, Madrid, Spain, December 1998.

[4] G. Buttazzo, E. Bini, and D. Buttle. Rate-adaptive tasks: Model, analysis, and design issues. In Proceedings of the International Conference on Design, Automation and Test in Europe (DATE 2014), Dresden, Germany, March 24-28, 2014.

[5] G. Buttazzo, G. Lipari, M. Caccamo, and L. Abeni. Elastic scheduling for flexible workload management. IEEE Transactions on Computers, 51(3):289-302, March 2002.

[6] D. Buttle. Real-time in the prime-time. Keynote speech given at the 24th Euromicro Conference on Real-Time Systems (ECRTS 2012), Pisa, Italy, July 12th, 2012.

[7] R. I. Davis, T. Feld, V. Pollex, and F. Slomka. Schedulability tests for tasks with variable rate-dependent behaviour under fixed priority scheduling. In Proceedings 20th IEEE Real-Time and Embedded Technology and Applications Symposium (RTAS 2014), Berlin, Germany, April 15-17, 2014.

[8] R. I. Davis, T. Feld, V. Pollex, and F. Slomka. Schedulability tests for tasks with variable rate-dependent behaviour under fixed priority scheduling. In University of York, Department of Computer Science Technical Report, YCS-2014-488, Jan, 2014.

[9] INTERESTED project partners. Interested, european project, cordis project description. URL: http://cordis.europa.eu/fp7/ict/embeddedsystems-engineering/factsheets/interested.pdf, 2008.

[10] K. Jeffay and D. Bennett. A rate-based execution abstraction for multimedia computing. In Proceedings of the Fifth International Workshop on Network and Operating System Support for Digital Audio and Video, Lecture Notes in Computer Science, SpringerVerlag, Vol. 1018, pages 67-78, Durham, NH, April 1995.

[11] K. Jeffay and S. Goddard. A theory of rate-based execution. In Proceedings of the 20th IEEE Real-Time Systems Symposium, pages 304-314, Phoenix, AZ, December 1999.

[12] J. Kim, K. Lakshmanan, and R. Rajkumar. Rhythmic tasks: A new task model with continually varying periods for cyber-physical systems. In Proceedings of the Third IEEE/ACM International Conference on Cyber-Physical Systems (ICCPS 2012), pages 2838, Beijing, China, April 17-19, 2012.

[13] C. Liu and J. Layland. Scheduling algorithms for multiprogramming in a hard-real-time environment. Journal of the Association for Computing Machinery, 20(1):46-61, January 1973.

[14] A. Mok. Fundamental Design Problems of Distributed Systems for the Hard Real-Time Environment. $\mathrm{PhD}$ thesis, Massachusetts Institute of Technology, Department of Electrical Engineering and Computer Science, Cambridge, MA, 1983.

[15] A. K. Mok and D. Chen. A multiframe model for real-time tasks. IEEE Transactions on Software Engineering, 23(10):635645, October 1997

[16] V. Pollex, T. Feld, F. Slomka, U. Margull, R. Mader, and G. Wirrer. Sufficient real-time analysis for an engine control unit with constant angular velocities. In Proc. of the Design, Automation and Test Conference in Europe, Grenoble, France, March 18-22, 2013.

[17] J. Real and A. Crespo. Mode change protocols for real-time systems: A survey and a new proposal. Real-Time Systems, 26(2):161-197, March 2004.

[18] L. Sha, R. Rajkumar, J. P. Lehoczky, and K. Ramamritham. Mode change protocols for priority-driven preemptive scheduling. RealTime Systems, 1(3):243-264, December 1989.

[19] M. Stigge and W. Yi. Combinatorial abstraction refinement for feasibility analysis. In Proceedings of the 34nd IEEE Real-Time Systems Symposium (RTSS 2013), 2013.

[20] N. Stoimenov, S. Perathoner, and L. Thiele. Reliable mode changes in real-time systems with fixed priority or EDF scheduling. In Proceedings of the Design, Automation and Test Conference in Europe (DATE 2009), Nice, France, April 20-24, 2009. 